\title{
The duration properties of Swift Gamma-Ray Bursts
}

\author{
Z.-B. Zhang ${ }^{1,2}$ and C.-S. Choi ${ }^{1}$ \\ ${ }^{1}$ International Center for Astrophysics, Korea Astronomy and Space Science Institute, \\ 36-1 Hwaam, Yusong, Daejon 305-348, South Korea; \\ email: z-b-zhang@163.com \\ ${ }^{2}$ Yunnan Observatory, National Astronomical Observatories, Chinese Academy of Sciences, \\ P. O. Box 110, Kunming 650011, China
}

\begin{abstract}
We report the systematic analysis of the durations for Swift gamma-ray bursts (GRBs) and compare the results with those of pre-Swift data. We show that the durations of Swift bursts also have two log-normal distributions that are clearly divided at $T_{90}=2 \mathrm{~s}$. Their intrinsic durations also show a bimodal distribution but shift systematically toward the smaller value compared with the observed one. This study confirms the spectra of short GRBs are in general harder than the long GRBs and shows that this trend becomes weak in the source frame.
\end{abstract}

Keywords. gamma-rays: bursts - gamma rays: theory

\section{Introduction}

Based on an analysis of durations using initial BASTE data, Kouveliotou et al. (1993) divided gamma-ray bursts (GRBs) into two classes, i.e., long GRBs (LGRBs) with $T_{90}>2 \mathrm{~s}$ and short GRBs (SGRBs) with $T_{90}<2$ s. Koshut et al. (1996) pointed out that the observed duration distribution may vary with instruments. It is therefore necessary to investigate if there exists a new GRB class and/or what physical factors produce such properties (Gehrels et al. (2004)). In this study, we focus on the relevant issues using the updated Swift data.

To study the intrinsic properties of GRBs, we selected six data sets, namely s1 - s6. As of 2007 July 1, Swift has detected 75 LGRBs (s1) and 20 SGRBs (s2) with known duration and redshift. 44 GRBs from s1 also have available $E_{p}$ values and constitute our sample s3. In s2, only 11 sources have a measured $E_{p}$ and are employed to build the sample s4. For the remaining 9 bursts in s2 without the measured redshifts, we assigned a redshift value of $z=0.5$ to the 9 bursts, approaching the median redshift of $z=0.4$, as assumed by Norris and Bonnell (2006). In our fifth sample set, s5, we include 48 preSwift LGRBs whose $z$ and $T_{90}$ are already measured, in which 18 sources, less than half $(\sim 38 \%)$ of the 48 pre-Swift bursts, are detected by the BATSE mission and constitute our sample s6. The detailed sample selection can be referred to Zhang \& Choi (2008).

\section{Results}

\subsection{Observed $T_{90}$ distribution}

Right panel in Figure 1 shows the $T_{90}$ distribution for the 95 Swift GRBs, which include s1 and s2 samples. The best fit with a two-lognormal function gives the center values $\left(T_{90, p 1}=0.28 \mathrm{~s}\right.$ and $\left.T_{90, p 2}=42.83 \mathrm{~s}\right)$ and the widths $\left(w_{1}=19.05 \mathrm{~s}\right.$ and $\left.w_{2}=18.20 \mathrm{~s}\right)$ with the reduced Chi-square $\chi^{2} /$ dof $=0.67$, which are roughly consistent with those calculated from the BATSE data (McBreen et al. 1994; Meegan et al. 1996; Paciesas et al. 1999; Horváth 2002; Nakar 2007). The superposed function has a minimum around $2 \mathrm{~s}$ as found by Kouveliotou et al. (1993), indicating that the Swift sources are also 

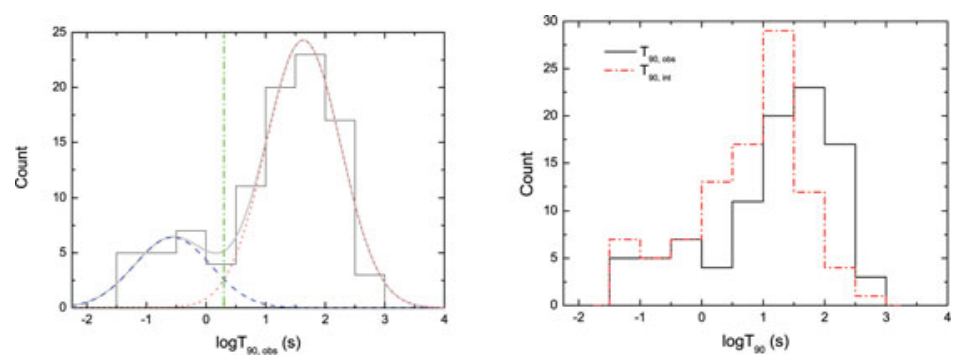

Figure 1. Left panel: shows the bimodal distribution of durations for the 95 GRBs (s1 and s2; histogram) and two-component log-normal fit to the data (solid line). The GRBs are divided into two classes at $T_{90} \simeq 2 \mathrm{~s}$ (vertical line): LGRBs (dotted line) and SGRBs (dashed line). Right panel shows a comparison between the observed (solid line the same as Fig. 1) and the intrinsic (dashed-dotted line) $T_{90}$ distributions.

divided into two classes, SGRBs and LGRBs, although the Swift is more sensitive to long soft bursts than the BATSE (Band 2006; Gehrels et al. 2007).

\subsection{Intrinsic $T_{90}$ distribution}

The increasing of the fraction of GRB sources with higher redshift may lead to an evident discrepancy of the intrinsic $T_{90}$ distributions between Swift and pre-Swift. The transformation of $T_{90}$ from observer frame to source frame is generally expressed as $T_{90, \text { int }}=T_{90} /(1+z)^{\omega}$, in which $\omega=1$ has been used, corresponding the case of no energy stretching. As shown in Figure 1 (right panel), the $T_{90, i n t}$ has a bimodal distribution and is significantly shifted toward shorter durations than the observed one. The best fit with a two-lognormal function gives two centers $\left(T_{90, p 1}=0.13 \mathrm{~s}, T_{90, p 2}=12.30 \mathrm{~s}\right)$ and two widths $\left(w_{1}=10.96 \mathrm{~s}\right.$ and $\left.w_{2}=17.38 \mathrm{~s}\right)$ with $\chi^{2} /$ dof $=0.92$, indicating that the distribution of $T_{90, \text { int }}$ is indeed bimodal but systematically narrower and shifted towards low values of duration in comparison to the observed one.

\section{Conclusions}

We find that Swift $T_{90}$ have two-lognormal distributions divided clearly at $T_{90} \simeq 2 \mathrm{~s}$. This implies that the classification in terms of duration is unchanged from pre-Swift to the Swift era. Swift $T_{90, i n t}$ also show a bimodal distribution but shifted systematically toward the smaller value and the distribution exhibits a narrower width relative to the observed one. In addition, the trend of LGRBs with a relatively softer spectrum largely weakens in the source frame, relative to SGRBs.

\section{References}

Balázs, L. G., et al. 2003, A\&A, 401, 129

Band, D. L. 2006, ApJ, 644, 378

Gehrels, N. et al. 2004, ApJ, 611, 1005

Gehrels, N., Cannizzo, J. K., \& Norris, J. P. 2007, NJPh, 9, 37

Horváth, I. 2002, A\&\&A, 392, 791

Kouveliotou, C., et al. 1993, ApJ 413, L101

Koshut, T. M., et al. 1996, ApJ, 463, 570

McBreen, B., Hurley, K. J., Long, R. et al. 1994, MNRAS, 271, 662

Meegan, C. A., et al. 1996, ApJS, 106, 65

Nakar, E. 2007, PhR, 442, 166

Norris, J. P. \& Bonnell, J. T. 2006, ApJ, 643, 266

Paciesas, W., Meegan, C., Pendleton, G., et al. 1999, ApJS, 122, 465

Zhang, Z. B. \& Choi, C. S. 2007, A\&GA, 484, 293 